\title{
ANALYSES OF DIFFERENT PROTOCOLS FOR MULTIPATH ROUTING IN WSN
}

\author{
Dr Suseela B, \\ Department of Electronics and Communication Engineering, \\ Government Polytechnic College, \\ Keelakanavai, Perambalur, Tamil Nadu, India \\ susikarikalan@gmail.com
}

\begin{abstract}
Modern progress in Wireless Sensor Networks (WSNs) for instance observation, traffic observation as well as storeroom of probable applicable are planned to cherish the authentic planet. For these network severe necessities of quick, dependable protocol to convene the restraint that is latency, highest packet received ratio, good put as well as energy efficiency. In this article we have reviewed the several multi path routing protocols such as Energy Efficient Multi Path (EEMP) routing protocol, Secure and Energy efficient Multi Path (SEMP) routing protocol, Bandwidth Aware Multi Path (BAMP) routing protocol, Cooperative Multi Path (CMP) routing protocol as well as QoS Multi Path (QoSMP) routing protocol. The simulation of multi path routing is offered depend on packet received ratio, average delay as well as energy efficiency in WSN.

Keywords: Energy Efficient Multipath Routing, Secure and Energy Efficient Multipath Routing, Cooperative Multi path Routing, Bandwidth Aware Multipath Routing, QoS Multi path Routing, wireless sensor network
\end{abstract}

\section{INTRODUCTION}

Usually WSN is spread for a broad series of applications, for instance clever moving, armed forces, as well as resident fields. The features of sensor node are least expenditure, easiness, as well as transmit, have additional stimulated the preparations of WSNs [1]. The WSN, have great amount of nodes as well as the coverage might not be, comprise to collect the information from still neglected position as well as communicate the information to the target, for such transaction of information enforces option of route that assures the information obtained ratio, least delay as well as target restricted available [2]. Figure 1 illustrates the example figure of WSN.

The collection of route decreases into two classes namely single path otherwise Multi Path (MP). Commonly, MP offers number of routes for assembly, the travel can be dividing as well as forward via the dissimilar routes thus attain superior load assessment can be reached except in MP system. Although it will induces delay as well as queuing latency whilst communicating the information concurrently. Thus that displace route in multiple path is preferred thus minimize the Routing Overhead (RO) by negligible path finding. Therefore it can attain superior load equilibrating as well as get better the pliability to collapse [3].

MP supportive path forwards packets in information flowing alongside multi-path concurrently, hence can get better good put except latency considerably. The categorization of packet communication between MPs, though, outcomes in elevated overhead on bandwidth as well as period. MP routing is a proficient method to utilize system resources with offering numerous routes among sender as well as target [4]. 
In this article, different multi path routing protocols in WSN in terms of Packet Received Rate (PRR), Average Delay (AD) as well as Remaining Energy (RE are examined. The organization of this article is as follows: Section 2 explains different multi path routing protocols in WSN and their performances in terms of $\mathrm{PRR}, \mathrm{AD}$ and RE are explained in section 3. Finally, conclusion is made in the last section.

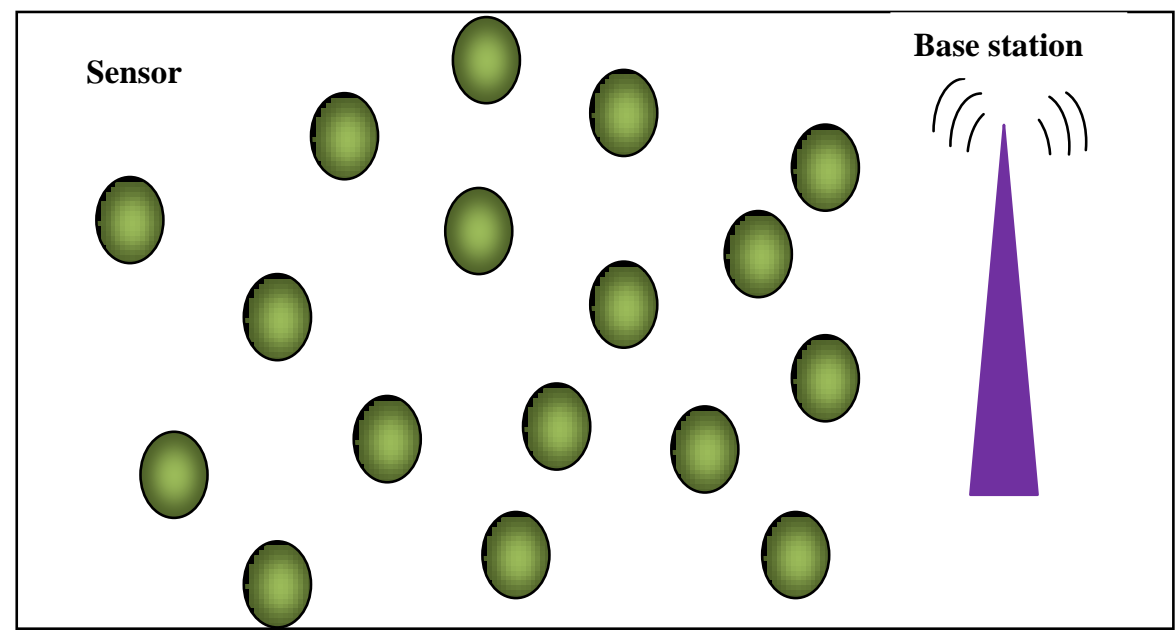

Fig. 1 Wireless Sensor Network

\section{METHODS AND MATERIALS}

Generally, two types of routing protocols are available namely single as well as MP routing protocols. Single path represents the only one routing path is available from sender to target in the network. Figure 2 illustrates the single path route in the network.

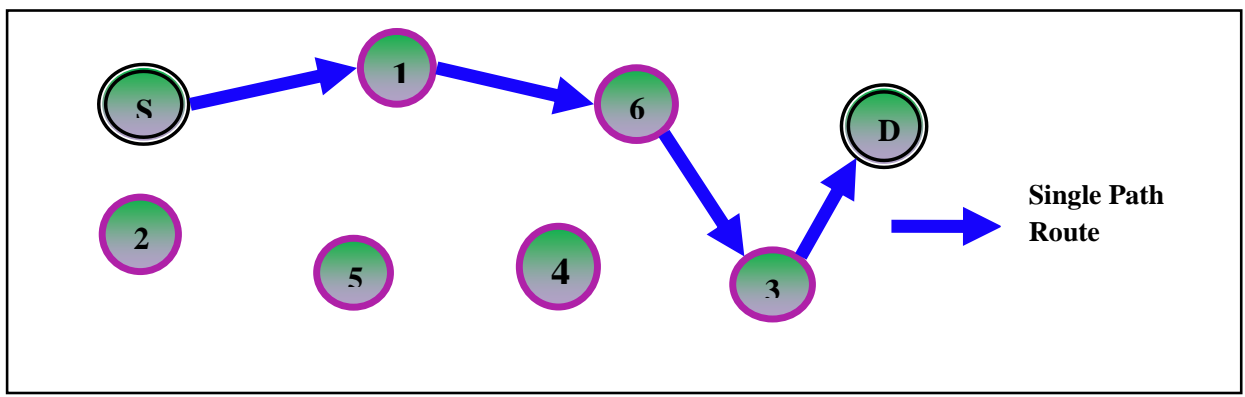

Fig. 2 Single path routing

The important properties of MP protocols are numerous routes as well as loop-free path which is shown in Fig. 3. In MP routing, discovers amount of several paths to the target however it has dual restraints. Initially, sensor node entered in one route could enter in other route name as disjointness. Sensor Node entered in route could not enter again in the similar route known as loop free.

In WSN initial route, such as the route with least delay. Main path may be selected depend on the route of cost otherwise hop, etc. Residual routes represent 
the interchange route that may be a little less than the main route. But the exchange routes with extended delay are less wanted. The disjointness minimizes the route belief of every other, thus route crash happens in one path leftovers nearby might not concern the other route [5].

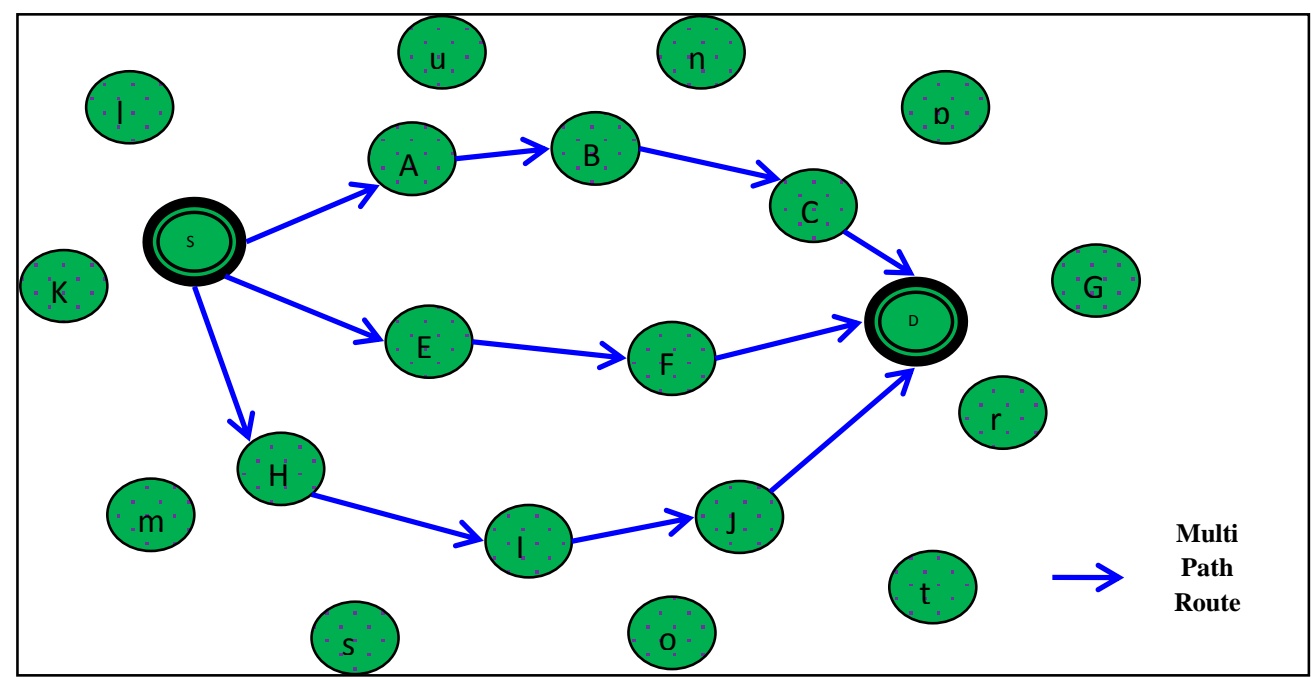

Fig. 3 Multi path routing

MP routing is an effectual approach to attain strength, load equilibrating, overcrowding decrease, as well as little energy expenditure. Disjoint MP routing offers amplified safety as well as bandwidth equated to non disjoint MP routing as connection- or else node-disjoint routes are applied. Although encourage in MP path explore, utilize of MP routing in IP systems is mainly imperfect to equal-cost MPs [6].

\section{A. EEMP Routing Protocol}

Energy expenditure is regarded as the significant restrictions in WSN since the sensor nodes cannot acquire stable energy provide as well as trust on batteries accordingly, minimizing lifetime because batteries obtain shattered extremely. In EE-MP, employing the Fitness Function procedure (FFP) is utilized to minimize the energy expenditure in MP routing protocol. This fitness occasion is assist to discover the better route from the sender to the target thus diminishes the energy expenditure in MP.

The FFP is an efficient method which gets as a fraction of several better methods for instance firefly method, genetic algorithm, particle swarm optimization (PSO) as well as bee colony method. The FFP discovers the significant issue in the better procedure that might be several components. The PSO algorithm is used to compute the node fitness function. The FFP computation components are distance, latency, bandwidth, as well as energy. This FFP is utilized for optimizing the different path in case the most important path breaks [7].

The PSO algorithm is formatted with residents of arbitrary applicant results, conceived as particles. Every particle is allotted an arbitrary velocity as well as iteratively via the space. It is concerned for the position of the better condition attained therefore faraway with the particle itself as well as with the position of the better fitness reached thus faraway diagonally the entire residents.

The PSO algorithm contains several alteration factors that significantly 
manipulate the algorithm act, frequently stated as the examination trade-off. Examination is the capability to analysis several areas in the trouble space to position a high-quality best, confidently the overall one. Development is the capabilities to deliberate seek around a hopeful applicant key to position the best exactly. In this event, the particles are concerned for two fitness factors namely energy level as well as distance. The sender transmits the packet via least energy as well as minimum distance thus enhances the route optimization.

\section{B. SEMP Routing Protocol}

The MP with secret sharing is extensively predictable as the valuable routing schemes to make sure the security entropy. This class of method translates every packet keen on numerous contributions to improve the safety of communication. This secret-sharing MP routing objective is improving both safety as well as lifetime. The secret-sharing algorithm that disseminative as well as arbitrarily offers contributions entire network [8].

This scheme offers security as well as lifetime. In this protocol, the threshold safety apportioning that disseminates in a definite area about the sender node. Next, deals an arbitrarily transmitted next to the same hop paths over the entire system. At last, this scheme communicates to the target node with utilizing smallest amount hop routing. The packet prevention possibility of this protocol is received in individual as well as multi black-hole cases also the smallest necessary size of the actually protected field is copied.

This scheme mainly concentrates on growing protection with the applying accessible energy to transmit shares by MP routes. This protocol contains three phases namely Area disseminative routing, disjoint indistinguishable hop routing as well as to the lowest degree hop routing. This protocol attempts to raises the assortment of paths with transmitting shares to the nodes depressively disseminated system with displace paths. As a result, the area disseminative paths as well as disjoint indistinguishable hop path degrees objective at arbitrarily disseminating the shares in the system as well as next communicates the shares to the target with the smallest amount hop routing. The safety as well as energy efficiency based multipath routing method to take full advantage of both the safety as well as the lifetime.

\section{BAMR Routing Protocol}

This routing protocol is capable to efficiently minimize the energy utilization through multi node cooperation as well as reserve allotment. The BAMR using a polynomial-time heuristic algorithm is utilized for resolve the transmitter obligation as well as power allotment. The objective of BA-MR is assist to reduce the power utilization of MP routing. This protocol contains two concurrent procedures such as MP route production as well as cooperative relay. These two methods make energy proficient node disjoint helpful MP routing thus enhance the efficiency of bandwidth [9].

Regarding a MP that contains a sender, target as well as transmitter nodes also it establish its NP- severity with decrease from helpful data collection difficulty. In this scheme, a polynomial-time heuristic algorithm for discovering least weight, disjoint paths since sender to target on a weighted graph that solve the bandwidth requirement [10]. Active training is utilized for decide forwarder task as well as energy allotment on the gained routes. This scheme builds energy proficient node disjoints MP routing whilst fulfilling the bandwidth restraint on every route. 


\section{CMP Routing Protocol}

Cooperative transaction is a suitable mechanism for enhance the special multiplicity exclusive of extra antennas. MP routing be able to mainly enhance the good put as well as minimize the latency. Network coding based MP routing dispatches the difficult categorization between MPs thus improves the information communication proficiency [11].

In this scheme, Non break Wait ACK (acknowledgement) method is used to alleviate the RO induced with waiting ACK messages. This scheme uses a sourcebased method as well as destination-based method for enhances the performance. This protocol obtain how much packets in a group could transmit for productive decrypting as well as additional forecast the communication periods which will be retransmitted in a possibility method. The CMPR can considerably enhance the good put as well as reduces the latency.

\section{E. QoSMP Routing Protocol}

The QoSMP routing protocol offers an assurance loop release, at the charge of effectiveness. This protocol is a competent as well as accurate hop-by-hop MP routing technique, which confirms several QoS parameters. This protocol used to assist a router in a link state system discovers numerous next-hops for every target. This protocol intend twice QoSMP routing methods known as MP method as well as lexico graphic MP, these competence is specially modified along with the isotonicity of the consequent mathematics, as well as these rightness is officially established [12].

Based on the routing metric algebra, two distributed multipath QoS routing algorithms are introduced to help a router in a link state network to find multiple next-hops for each destination. They are specifically tailored for algebras with strict or non-strict isotonicity, and their correctness is formally proved. In hop-by-hop routing, every node separately calculates its next-hops for the entire target. This protocol calculates the weakest route tree in a method like to the Dijkstra's rule.

\section{RESULTS AND DISCUSSION}

The routine of the EEMP, SEMP, BAMP, CPM and QoSMP are examined with utilizing the Network simulator (NS-2.35). Here, 50 sensor nodes spread in the region $1000 \times 1000 \mathrm{~m}^{2}$. The sensor nodes are transaction with all other sensor nodes via user datagram protocol. The traffic is managed by constant bit rate. The performance of the EEMP, SEMP, BAMP, CPM and QoSMP protocols are examined by the factors like PRR, AD as well as RE. PRR is the fraction of amount of packets received to entire target to the amount of packets transmits by the sender node. Figure 4 shows the PRR of various protocols in WSNs. 


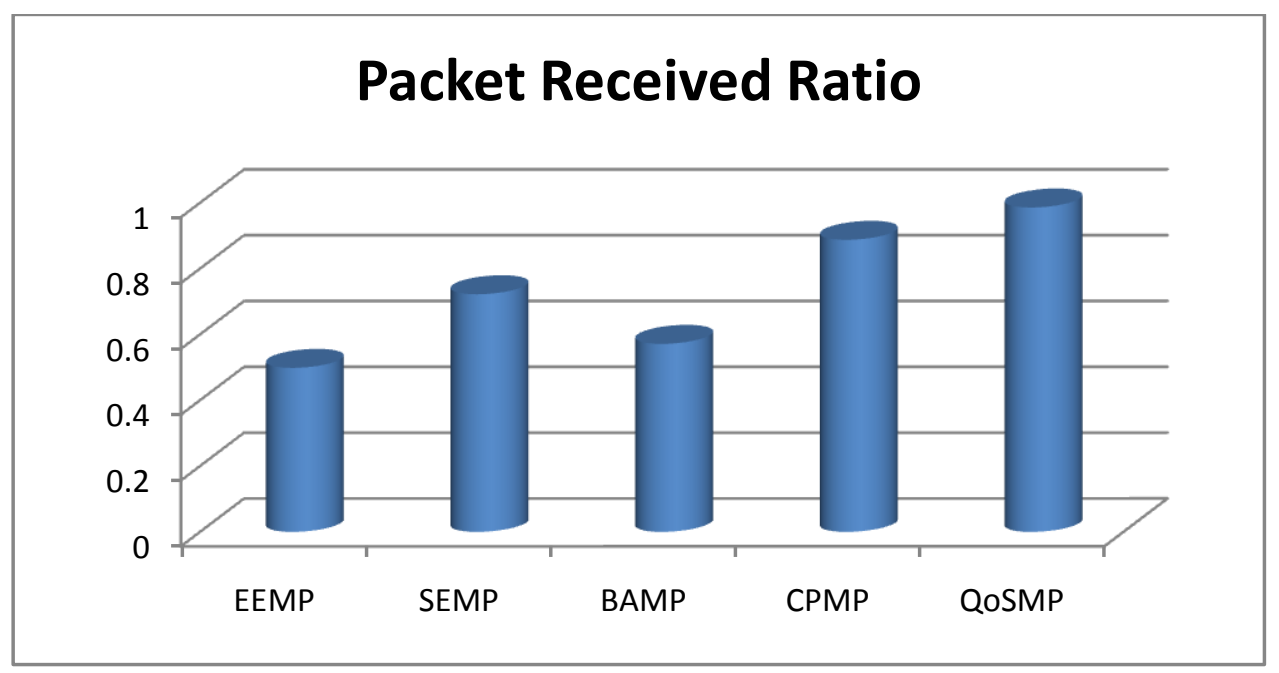

Fig. 4 PRR of EEMP, SEMP, BAMP, CPM and QoSMP

From Figure 4, it is observed that the PRR of QoSMP is higher than other protocols like EEMP, SEMP, BAMP, CPM because it considers the route based on the QoS. Thus it raises the PRR. The CPM has higher PRR than the EEMP, SEMP, BAMP except QoSMP protocol because it cooperates the relay node during data transmission. As a result, it reduces the loss of data packets. The SEMP has higher PRR than BEMP as well as EEMP but the PRR is lesser than QoSMP as well as CPM because it offers the security and energy proficiency. The EEMP has greater $\mathrm{AD}$ than all other routing protocols. The AD represents the time distinction among the present packets obtained as well as the earlier packet obtained. Figure 5 shows the AD of various protocols in WSNs.

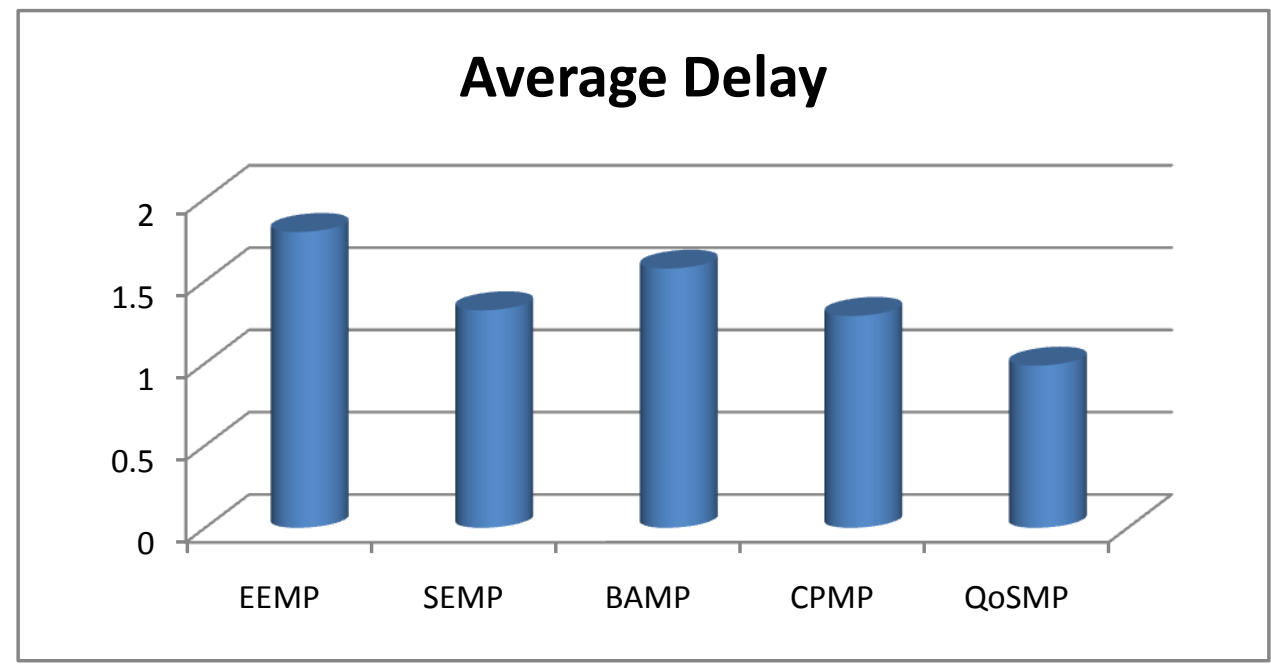

Fig. 5 AD of EEMP, SEMP, BAMP, CPM and QoSMP

From Figure 5, it is observed that the AD of QoSMP is lesser than the other protocols like EEMP, SEMP, BAMP, CPM because it considers the route based on the QoS. Thus it minimizes the AD. Then CPM has lesser AD than the EEMP, 
SEMP, BAMP except QoSMP protocol because it cooperates the relay node during data transmission. The SEMP has lower AD than BEMP as well as EEMP but the $\mathrm{AD}$ is greater than QoSMP as well as CPM. The EEMP has greater AD than all other routing protocols.

Energy proficient path protocols are proposed to improve the sensor energy levels. If diminish in power they turn into unable to transmit the packets. Such types of protocols are prepared to improve this energy between the sensor nodes. The amount of energy remaining in a node at the current instance of time is called as residual energy. Figure 6 shows the RE of various protocols in WSNs.

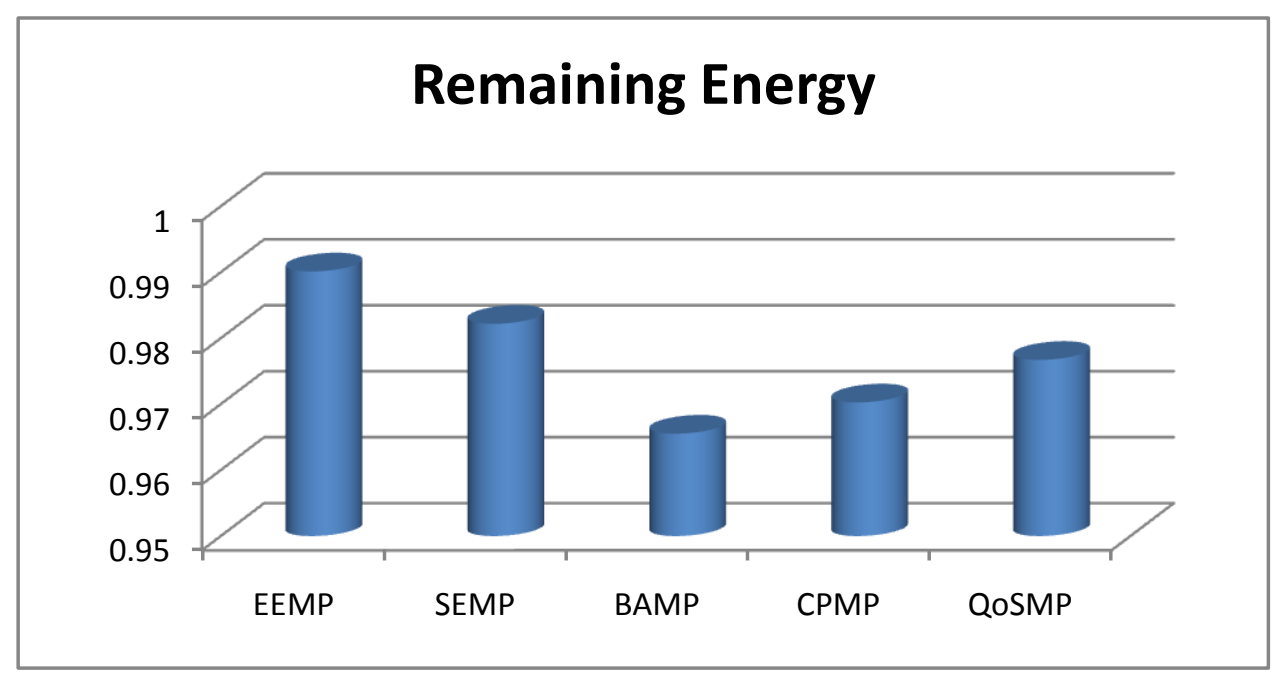

Fig. 6 RE of EEMP, SEMP, BAMP, CPM and QoSMP

It is inferred from Figure 6 that the RE of EEMP is superior to other protocols like SEMP, BAMP, CPM and QoSMP since it is highly significant for energy parameter. Next the SEMP is slightly lesser than the EEMP protocol because of the SEMP protocol main objective is to enhance the energy as well as security. The QoSMP has greater RE than BAMP as well as CPM. But, the RE of CMP is very low compare to all other protocols since this protocol not priority to the energy efficiency.

\section{CONCLUSION}

This study compares the function as well as simulation of MP routing protocols in WSN include packet received ratio, average delay as well as residual energy. In EEMP, the fitness function is used to calculate the energy efficiency. The SEMP routing protocol translates to improve the safety of communication as well as energy efficiency. The BAMP routing protocol builds energy proficient node disjoints MP routing whilst fulfilling the bandwidth restraint on every route. The CMP can considerably enhance the good put as well as reduces the latency. In QoSMP is an accurate hop-by-hop MP routing technique that confirms several QoS parameters. The simulation results illustrates the QoSMP perform better PRR as well as least delay. Also, EEMP enhances the energy efficiency than other MP routing protocols in the network. 


\section{REFERENCES}

[1]. J. He, and J. Rexford, "Toward internet-wide multipath routing. IEEE network", Vol. 22, No. 2, 2008, pp. 16-21.

[2]. T.M. Conte, U.S. Patent No. 8,582,502. Washington, DC: U.S. Patent and Trademark Office, 2013.

[3]. S.J. Lee, and M. Gerla, "Split multipath routing with maximally disjoint paths in ad hoc networks", IEEE International Conference on Communications, 2001, pp. 3201-3205.

[4]. M.A. Salkuyeh, and B. Abolhassani, "An adaptive multipath geographic routing for video transmission in urban VANETs", IEEE Transactions on Intelligent Transportation Systems, Vol. 17, No. 10, 2016, pp. 2822-2831.

[5]. A. Gopalan, and S. Ramasubramanian, "IP fast rerouting and disjoint multipath routing with three edge-independent spanning trees", IEEE/ACM Transactions on Networking, Vol. 24, No. 3, 2015, pp. 13361349.

[6]. D. Han, and J.M. Chung, "Self-similar traffic end-to-end delay minimization multipath routing algorithm", IEEE Communications Letters, Vol. 18, No. 12, 2014, pp. 2121-2124.

[7]. A. Taha, R. Alsaqour, M. Uddin, M. Abdelhaq, and T. Saba, "Energy efficient multipath routing protocol for mobile ad-hoc network using the fitness function", IEEE access, Vol. 5, 2017, pp. 10369-10381.

[8]. A. Liu, Z. Zheng, C. Zhang, Z. Chen, and X. Shen, "Secure and energyefficient disjoint multipath routing for WSNs", IEEE Transactions on Vehicular Technology, Vol. 61, No. 7, 2012, pp. 3255-3265.

[9]. H. Xu, L. Huang, C. Qiao, Y. Zhang, and Q. Sun, "Bandwidth-power aware cooperative multipath routing for wireless multimedia sensor networks", IEEE Transactions on Wireless Communications, Vol. 11, No. 4, 2012, pp. 1532-1543.

[10]. Y. Ganjali, and A. Keshavarzian, "Load balancing in ad hoc networks: single-path routing vs. multi-path routing:, In IEEE INFOCOM, 2004, pp. $1120-1125$.

[11]. F. Tang, H. Zhang, and L.T. Yang, "Multipath Cooperative Routing with Efficient Acknowledgement for LEO Satellite Networks", IEEE Transactions on Mobile Computing, Vol. 18, No. 1, 2018, pp. 179-192.

[12]. H. Geng, X. Shi, X. Yin, Z. Wang, and S. Yin, "Algebra and algorithms for multipath QoS routing in link state networks" Journal of Communications and Networks, Vol. 19, No. 2, 2017, pp. 189-200. 